\title{
El gasto social como contención de la población obrera sobrante durante el kirchnerismo y el chavismo (2003-2010)
}

\author{
Social Spending as a Control of the Unemployed Working Class \\ during Kirchnerism and Chavism (2003-2010)
}

Tamara Seiffer

Instituto de Investigaciones Gino Germani (IIGG)

Universidad de Buenos Aires

tamara_seiffer@yahoo.com.ar;

Juan Kornblihtt

Instituto de Estudios de América Latina y El Caribe (IEALC)

Universidad de Buenos Aires

jkornblihtt@gmail.com;

Romina de Luca

Centro de Estudios e Investigación en Ciencias Sociales (CEICS)

Universidad de Buenos Aires

rom.deluca@gmail.com

Recibido: 28/10/2011

Revisado: 21/11/2011

Aceptado: 01/12/2011

Disponible on line: 15/02/2012

\section{Resumen}

La última década se caracteriza por un fuerte aumento del gasto social en América Latina. Esto se observa en los casos de Argentina y Venezuela, donde el fenómeno se explica por la presencia de gobiernos llamados «nacional-populares» o «socialistas». Según afirman numerosos autores, estaríamos frente a un cambio cualitativo en relación a las políticas neoliberales adoptadas en la década de los años noventa. En este trabajo nos proponemos discutir este consenso y mostrar que el fuerte aumento del gasto social no responde a un cambio cualitativo motorizado por un giro a la izquierda de los gobiernos en cuestión, sino a un crecimiento cuantitativo dentro de una tendencia previa a la expansión de la acción estatal para contener a la creciente población obrera sobrante para el capital que se consolida en ambos países. Palabras clave: Política social, Gasto social, Sobrepoblación relativa, Asistencia social, Acumulación de capital

\begin{abstract}
The last decade is marked by a strong rise in social spending in Latin America. This is particularly observed in Argentina and Venezuela, where this phenomenon was explained by the presence of so-called «national and popular» or «socialist» governments. Many authors maintain that we are facing a qualitative change from the neoliberal politics of the ' $90 \mathrm{~s}$. In this paper, we propose to discuss this consensus by showing that the strong rise of social spending does not express a qualitative change propelled by a turn to the left but rather to a quantitative expansion, following a prior trend of the State's role in order to control the rising unemployed, or surplus, working class population for the capital that is being consolidated in both countries.
\end{abstract}

Keywords: Social policies, Social spending, Surplus population (the Unemployed), Social welfare, Capital accumulation

Referencia normalizada: Seiffer, R.; Kornblihtt, J. \& De Luca, R. (2012): «El gasto social como contención de la población obrera sobrante durante el kirchnerismo y el chavismo (2003-2010)». Cuadernos de Trabajo Social, 25(1): $33-47$.

Sumario: Introducción. 1. La particularidad de la política social en la década de los años 2000. 2. La consolidación de la población sobrante. 3. Política social y capital sobrante. 4. Conclusiones. 5. Referencias bibliográficas. 


\section{Introducción}

La última década pareciera abrir una nueva etapa en América Latina. Existe cierto consenso en que del «neoliberalismo» propio de los años 1990, marcado por la supuesta retirada del Estado, se habría pasado a una fuerte presencia del mismo en la economía y a un cambio en su rol, en particular a través de una fuerte transformación de la política social. Los casos de Argentina y Venezuela resultarían paradigmáticos: para el primero, el ascenso a la presidencia de Néstor Kirchner en 2003, sucedido luego por su esposa Cristina Fernández de Kirchner a partir de 2007. Ambos inaugurarían una nueva fase política artífice de desandar la «herencia neoliberal» y de cerrar la crisis política abierta en diciembre de 2001. A nivel local, dicha etapa se conoce como el ascenso del kirchnerismo y a ello nos referiremos en este artículo; por su parte, para el caso venezolano, la llegada a la presidencia de Hugo Chávez, en 1999, constituiría un correlato similar al del caso argentino. En Venezuela, el chavismo - nombre con el que se identifican a nivel político los partidarios de Hugo Chávez- sería el responsable de la reorientación estatal. Uno y otro se presentan como un cambio cualitativo en el contexto latinoamericano. Ambos son reconocidos por la importancia que ha tomado la política de asistencia social. El primero sustentado en los planteamientos de «desarrollo con inclusión social» $\mathrm{y}$ el segundo, en los que lo consideran vector de transformación de la estructura social hacia el «socialismo del siglo XXI» (Rodríguez Rojas, 2010; Reyes, 2006).

En ambos gobiernos, la población beneficiada por las políticas sociales se expande de manera notable. En el caso de Venezuela, entre 2003 y 2009 el Instituto Venezolano de Seguridad Social (IVSS) pasó de cubrir el 38 por ciento al 70 por ciento de la población: más de
19.000.000 venezolanos dependen actualmente de alguna forma de la asistencia estatal. En Argentina, la inexistencia de un padrón único de beneficiarios, dificulta la obtención de cifras claras de la cantidad de personas asistidas. Sin embargo, algunos datos pueden dar cuenta clara de este incremento: entre 2003 y 2011, las pensiones no contributivas aumentaron el 215 por ciento. Una estimación de beneficiarios de políticas de transferencia de ingresos, a partir de diversas fuentes, indica que tras una gran expansión en el año 2003, la cifra se estanca en 2.658 .000 beneficiarios promedio hasta 2008 y a partir de entonces crece, de forma exponencial, con la implementación de la Asignación Universal por Hijo $(\mathrm{AUH})^{1}$, alcanzando a más de 5.000.000 personas en $2011 .^{2}$

La expansión del sistema de asistencia se expresa, a su vez, en el gasto que el Estado destina a tal fin. Entre 2003 y 2009, Argentina y Venezuela duplican el poder adquisitivo del gasto social por habitante. El fuerte aumento en las variables analizadas afianza la hipótesis de un cambio cualitativo en la última década. Sin embargo, intentaremos mostrar que este incremento, posibilitado por el crecimiento económico de ambos países en un contexto de movilización de la clase obrera, no es algo novedoso ni en su evolución ni en su carácter. Para empezar, podemos ver que la tendencia a la expansión de las políticas sociales, como puede observarse en el gráfico 1, no es específica de esos gobiernos, sino que comienza en la década de 1990, con un crecimiento del gasto social promedio anual entre 1990 y el 2000 del 5,8 por ciento en Venezuela y del 3,6 por ciento en Argentina. La participación del gasto social en el PBI pasó del 10 por ciento al 16 por ciento en el primero y del 18 por ciento al 22 por ciento en el segundo. Puede destacarse que dentro de los ciclos generales de crecimiento, los momentos de disminución del

\footnotetext{
${ }^{1} \mathrm{La}$ AUH es un ingreso que se asigna a los hijos de desempleados y trabajadores no registrados y exige la vacunación y la asistencia escolar (ésta se incrementó un 1,6 por ciento). Pese a que representa una mejora sustancial de las condiciones de vida, no alcanza a cubrir una canasta básica de alimentación y, por lo tanto, no implica que la población que la reciba pueda reproducir su fuerza de trabajo en condiciones normales.

${ }^{2}$ La cifra está realizada en base a la suma de los beneficiarios de los programas de empleo del MTEySS (para el año 2010 no se poseen datos del Plan Jefes y Jefas de Hogar Desocupados, pero sí del Seguro de Capacitación y Empleo), beneficiarios de programas provinciales (hasta 2008), Programas dependientes del MDS (Pensiones no contributivas, Plan Familias por la inclusión social y Programa de ingreso social con trabajo), y titulares de la Asignación Universal por Hijo en los años 2009 y 2010.
} 


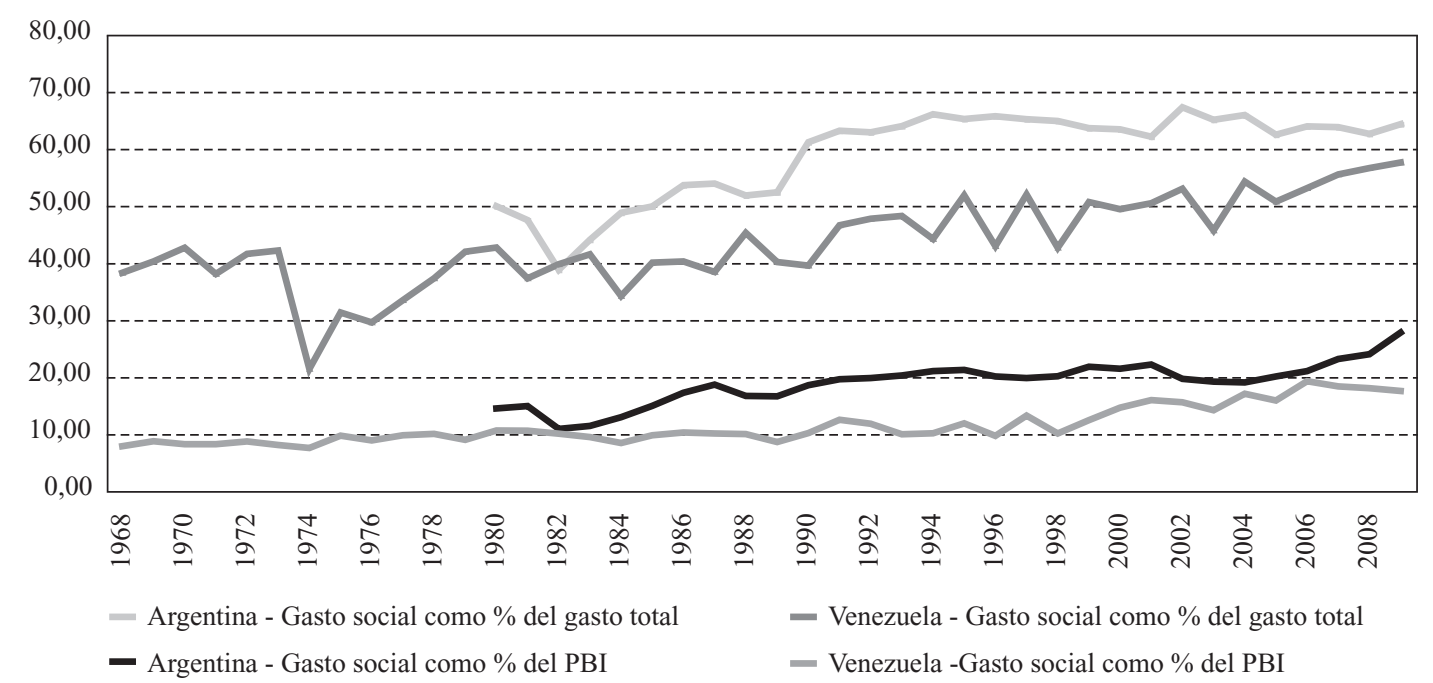

Gráfico 1. Gasto social como \% del PBI y del Gasto Total. Argentina (1980-2009), Venezuela (1968-2009). Fuente: elaboración propia en base a MECON y Aponte Blank (2006 y 2010).

gasto no coinciden con los gobiernos neoliberales sino con los períodos de crisis. En definitiva, la expansión del gasto tiene las mismas bases que el conjunto de la economía.

Dados estos primeros indicios que muestran cierta continuidad, surge la necesidad de ver si más allá de estos primeros datos cuantitativos estamos frente a un cambio cualitativo. En ese sentido, las preguntas que guían este trabajo son ¿por qué se expande de tal forma la política social en Argentina y Venezuela? Esta ampliación ¿expresa un cambio cualitativo?

\section{La particularidad de la política social en la década de los años 2000}

En términos históricos, al comparar los dos países vemos que el gasto social ha sido más alto en el caso de Argentina, pero se observa en la última década una tendencia a la reducción de las distancias. Un elemento clave para entender esta reducción es que en ambos casos el gasto social está asociado a la evolución de los recursos disponibles por la apropiación estatal de la renta diferencial de la tierra. En el caso argentino, dicha apropiación creció a partir de 2003 por la aplicación de impuestos a las exportaciones, en particular de la soja. Aunque expresa una riqueza importante, no llega a superar el 12 por ciento de la recaudación total. En Venezuela la apropiación de la renta por parte del Estado es mayor porque su producción se encuentra estatizada y porque el precio del petróleo subió más que el de los cereales y oleaginosas (base de la renta argentina). Esto explica por qué pese a ser una economía menor existen condiciones que posibilitan que el poder adquisitivo del gasto social venezolano en la última década reduzca su brecha con el argentino.

Rastreadas las condiciones para el fuerte aumento del gasto social, veamos su contenido en cada país. En el caso de Venezuela, el gasto se divide en seis rubros y en el de Argentina en ocho que, a su vez, tienen un total de nueve subrubros. A fines de poder realizar una comparación entre países hemos reacomodado los rubros y subrubros del gasto social argentino bajo las categorías del gasto venezolano tal y como puede verse en la tabla 1.

En cuanto a su distribución interna, en el caso de Argentina, es el rubro Seguridad Social el que ha tenido el lugar preponderante dentro de la estructura del gasto social, seguido por Educación. La serie permite observar una disminución relativa de estos rubros por la importancia que empiezan a tener otros rubros en el gasto social. Se destaca, a partir del año 2001, el peso relativo mayor que empieza a tomar Desarrollo y Participación, destinado a asistencia social (fundamentalmente planes alimenta- 


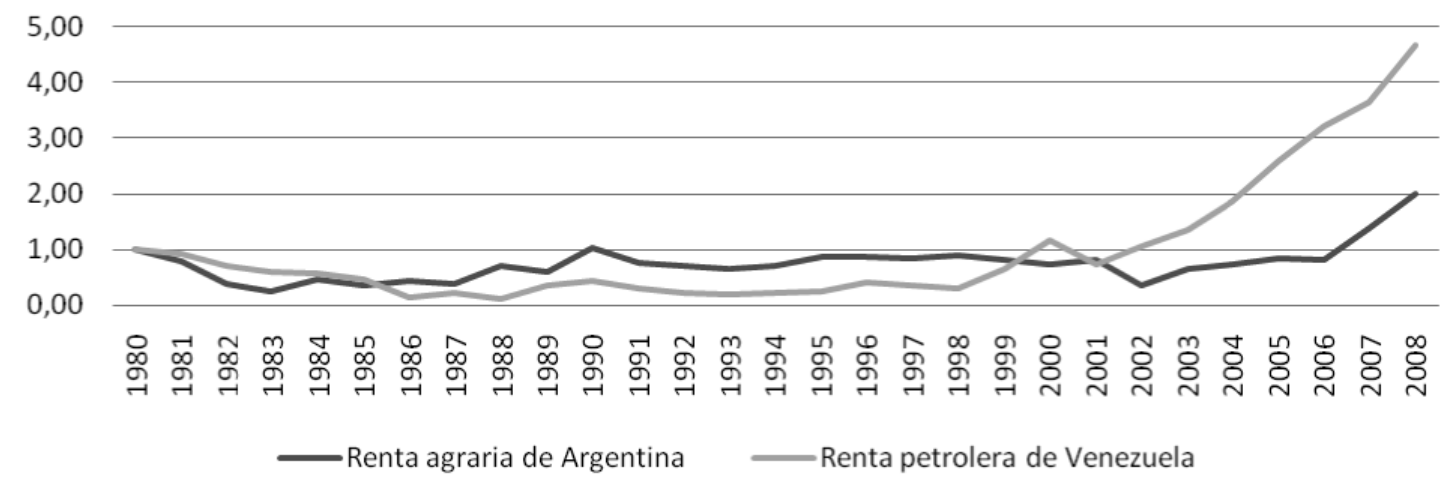

Gráfico 2. Evolución de la renta. Argentina y Venezuela $(1980=1)$. Fuente: elaboración propia en base a Íñigo Carrera (2007), Dachevsky (2011).

\begin{tabular}{|l|l|}
\hline \multicolumn{1}{|c|}{ Venezuela } & \multicolumn{1}{c|}{ Argentina* } \\
\hline Educación & Educación básica y Educación superior y universitaria \\
\hline Salud & Atención pública de la salud \\
\hline Vivienda & Vivienda y urbanismo \\
\hline Seguridad Social & $\begin{array}{l}\text { Obras sociales (atención de la salud y prestaciones sociales), INSSJyP } \\
\text { (atención de la salud y prestaciones sociales) y Asignaciones familiares }\end{array}$ \\
\hline Desarrollo y Participación & $\begin{array}{l}\text { Promoción y asistencia social pública y Programas de empleo y segu- } \\
\text { ro de desempleo }\end{array}$ \\
\hline Cultura y Comunicación & Cultura y Educación y cultura sin discriminar \\
\hline Ciencia y Técnica & Ciencia y Técnica \\
\hline$*$ Quedaron como residuales: el rubro «Otros servicios urbanos» y el sub-rubro «Agua potable y alcantarillado» \\
\hline
\end{tabular}

Tabla 1. Rubros del gastos social.

rios y planes de transferencia de ingresos). El gasto en Educación se mantiene relativamente estable pero, por la disminución proporcional del gasto en Seguridad Social, pasa a ser el primer componente del gasto.

En Venezuela, en cambio, el gasto en Educación ha significado históricamente la mayor proporción del gasto social, mientras que Seguridad Social ha cumplido un rol relativamente marginal. Pero en la última década este rubro, que es el que absorbe gran parte de la política asistencial en Venezuela, ha cobrado mayor importancia relativa, llegando a ocupar el segundo lugar del gasto social tal como se observa en el gráfico 4.

En Argentina, a pesar de los cambios en las proporciones del gasto, excepto los rubros de
Vivienda y Ciencia y Técnica, todos han tendido a crecer a lo largo de la serie. En el caso de Seguridad social y Educación, se observa una disminución relativa durante los años 1990 que encuentra su punto más bajo en el año 2002, a partir del cual crecen ubicándose por encima de los valores de la década de 1980. A partir del año 1997, Seguridad Social cede el primer lugar del gasto a Educación, tendencia que se mantiene presente hasta hoy. Y desde el año 2002 hasta el 2007, el gasto en Desarrollo y Participación supera al realizado en Salud, con el crecimiento relativo más importante: del 264 por ciento entre 1980 y 2009 y del 109 por ciento en la última década. Esta evolución puede observarse en el gráfico 5, donde se presentan los datos de los principales rubros del gasto. 


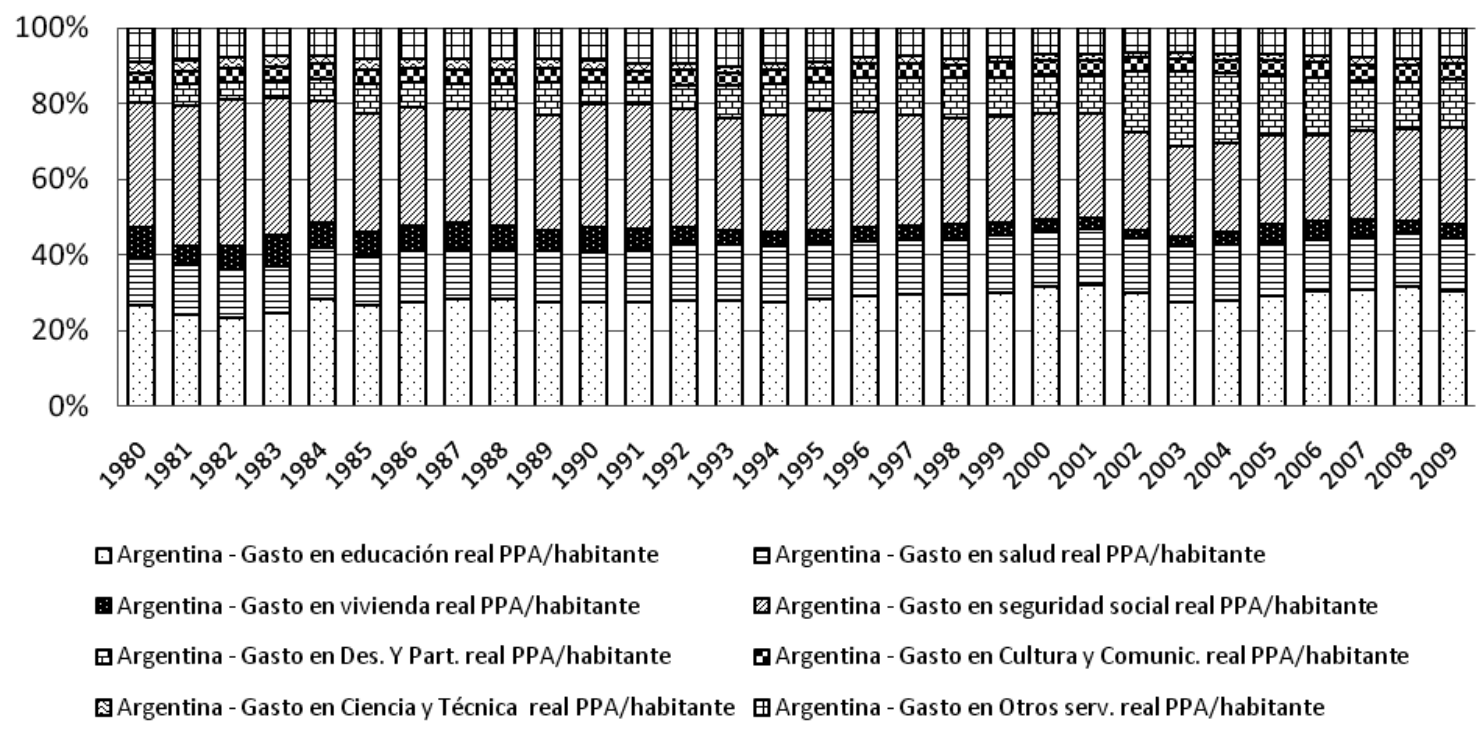

Gráfico 3. Rubros del gasto social como porcentaje del Gasto Social Total. Argentina (1980-2009). Fuente: Elaboración propia en base a MECON.

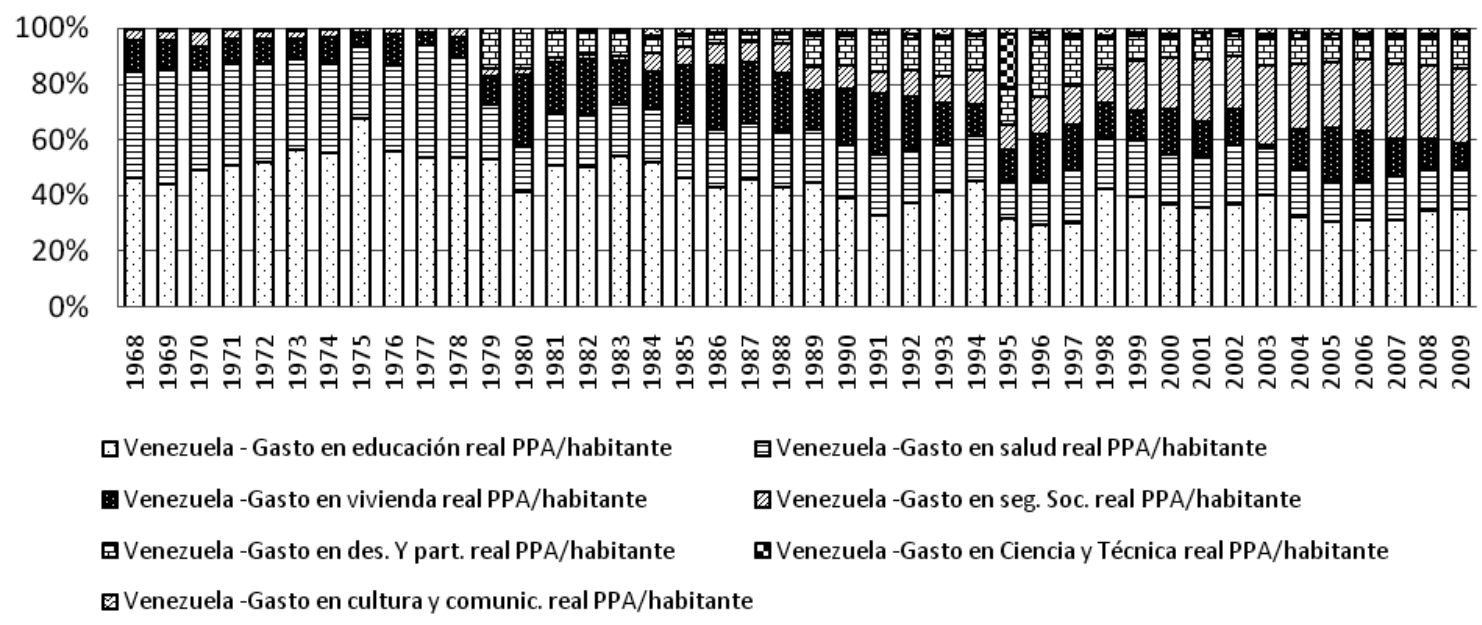

Gráfico 4. Rubros del gasto social como porcentaje del Gasto Social Total. Venezuela (1968-2009). Fuente: Elaboración propia en base a Aponte Blank (2006 y 2010).

En el caso de Venezuela, para lo cual se tiene en consideración una serie de gasto más larga, puede observarse, en primer lugar, la importancia del gasto en Educación y su caída desde mediados de los años 1970 hasta mediados de los años 1990, a partir de entonces se recupera llegando a superar los valores históricos. En segundo lugar, la importancia menor de Seguridad Social hasta fines de los años 1990 y su crecimiento exponencial durante la última década, supone ubicarse en el segundo puesto del gasto (con un crecimiento del 204 por ciento entre 1999 y 2009). Y, por último, el aumento del gasto en Desarollo y Participación en la última década (147 por ciento). Cabe destacar asimismo que los gráficos 5 y 6 permiten observar que el gasto social venezolano, así como sus diferentes destinos, es más fluctuante que el argentino.

$\mathrm{Si}$ analizamos el gasto en asistencia social por habitante, en términos de su poder adquisi- 


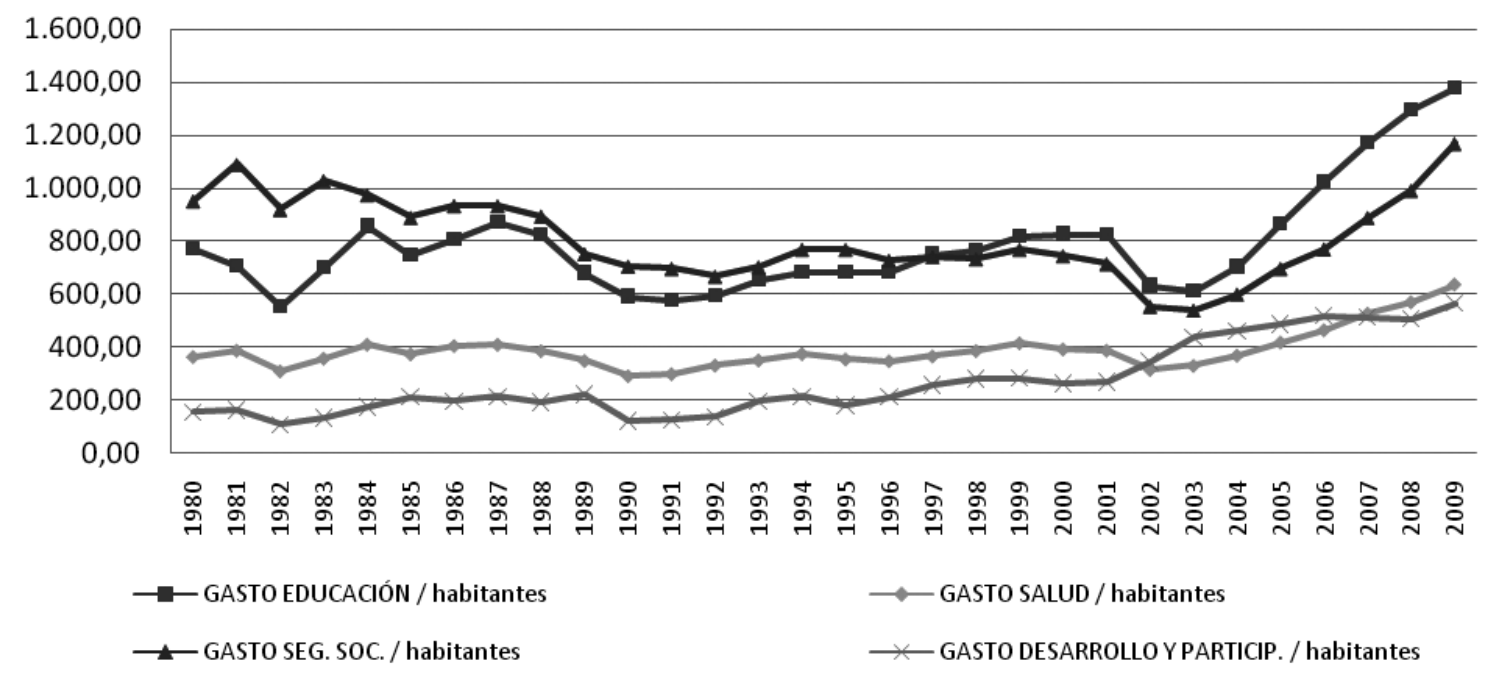

Gráfico 5. Principales rubros del Gasto social por habitante en \$ de 2008. Argentina (1980-2009). Fuente: elaboración propia en base a MECON.

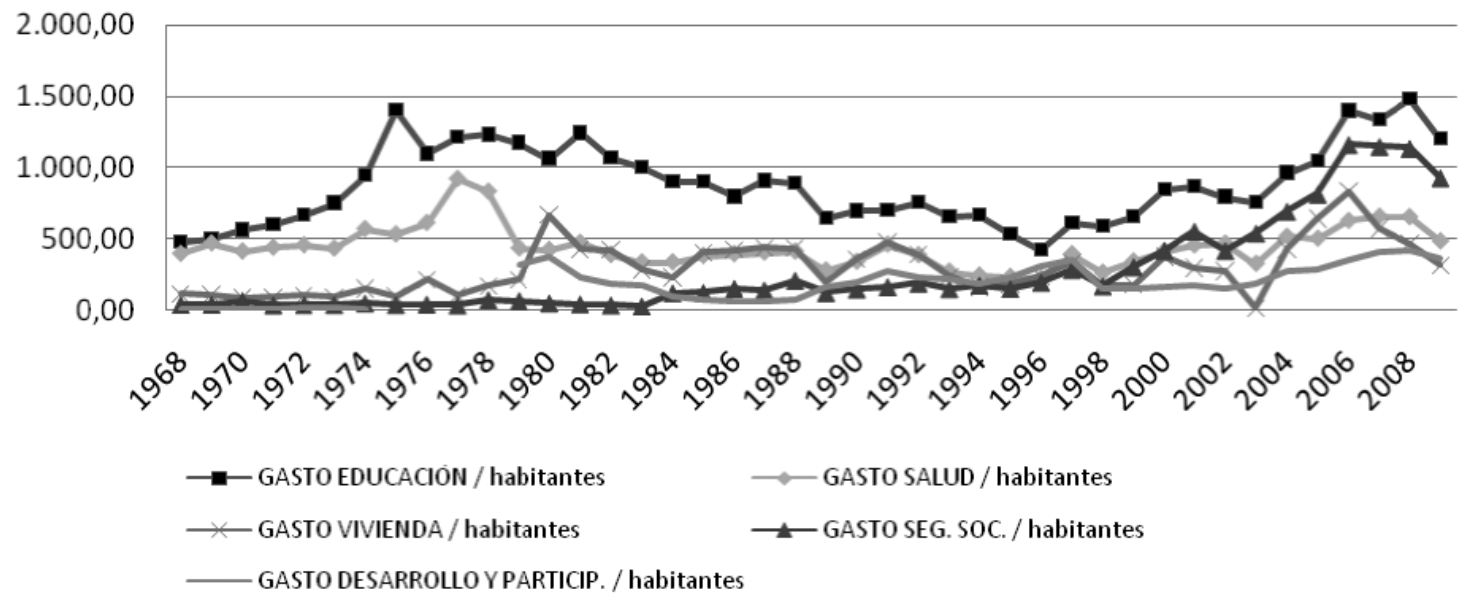

Gráfico 6. Principales rubros del Gasto social por habitante en BF de 2008. Venezuela (1968-2009). Fuente: elaboración propia en base a Aponte Blank (2006 y 2010).

tivo, hallamos que en Venezuela es mayor que en Argentina. Además, el Estado venezolano, a diferencia de Argentina hasta 2009, tiende a canalizar la mayor parte de la asistencia social a través del rubro de Seguridad Social. Si bien este rubro era relativamente marginal hasta fines de los años 1990, empieza a aumentar exponencialmente superando el poder adquisitivo del gasto realizado por el Estado argentino en la materia durante los años 2004 al 2007.
Argentina, durante esos años, expande su componente asistencial a través del rubro Desarrollo y Participación.

El análisis de la política asistencial del chavismo permite establecer dos períodos: el primero va desde su asunción en 1999 hasta 2002, en el que se mantiene la estructura y el nivel del gasto de décadas anteriores; el segundo se inicia en 2003 en donde el gasto se relanza. La segunda etapa se inicia con la aprobación, a 


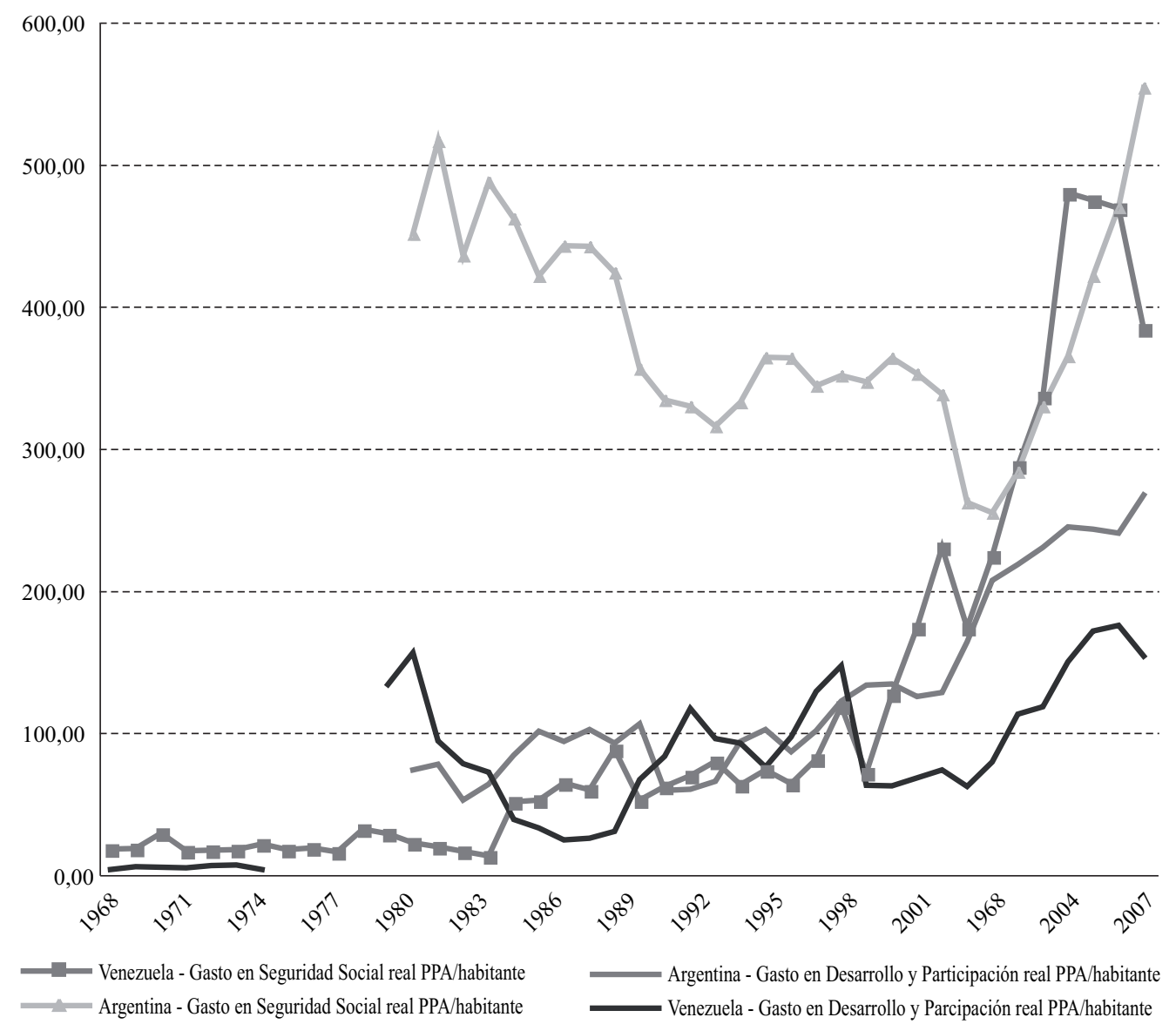

Gráfico 7. Gasto en Asistencia social por habitante en PPA de 1993. Argentina (1980-2009) y Venezuela (1968-2009). Fuente: elaboración propia en base a MECON y Aponte Blank (2006 y 2010).

finales de 2002, del anteproyecto de la Ley Orgánica del Sistema de Seguridad Social que propone garantizar el derecho de toda persona a recibir los beneficios de la seguridad social, con independencia de su ingreso y su financiación pública (Mateo Tomé, 2010). Se encuentra determinado también por la finalización del paro petrolero, en el primer trimestre de 2003, y por la derrota de la oposición en el referéndum revocatorio de 2004. Es, a partir de este año, cuando se observa un crecimiento del gasto social, que en su momento álgido supone más del doble de los valores previamente observados. En el caso argentino bajo el kirchnerismo logramos diferenciar tres momentos: el primero corresponde al inicio de su gestión caracterizada por un alto un nivel de conflictividad social y con la implementación masiva de un plan de transferencias condicionadas de ingresos como el Plan Jefes y Jefas de Hogar desocupados. ${ }^{3}$ El segundo se abre a mediados del año 2003, con la merma del nivel de conflictividad social. En esta segunda etapa se ponen en marcha una serie de planes que buscan desactivar el Plan Jefes y Jefas fragmentando a la población beneficiaria: el «Plan Familias por la Inclusión Social», el «Seguro de Capacitación y Empleo» y el Plan «Manos a la obra». La mayor fragmentación no implica una disminución del gasto en la materia. El ter-

${ }^{3}$ El mismo llegó a alcanzar a casi 2.000.000 trabajadores, se dirigía a Jefes de Hogar Desocupados y exigía una contraprestación laboral. 


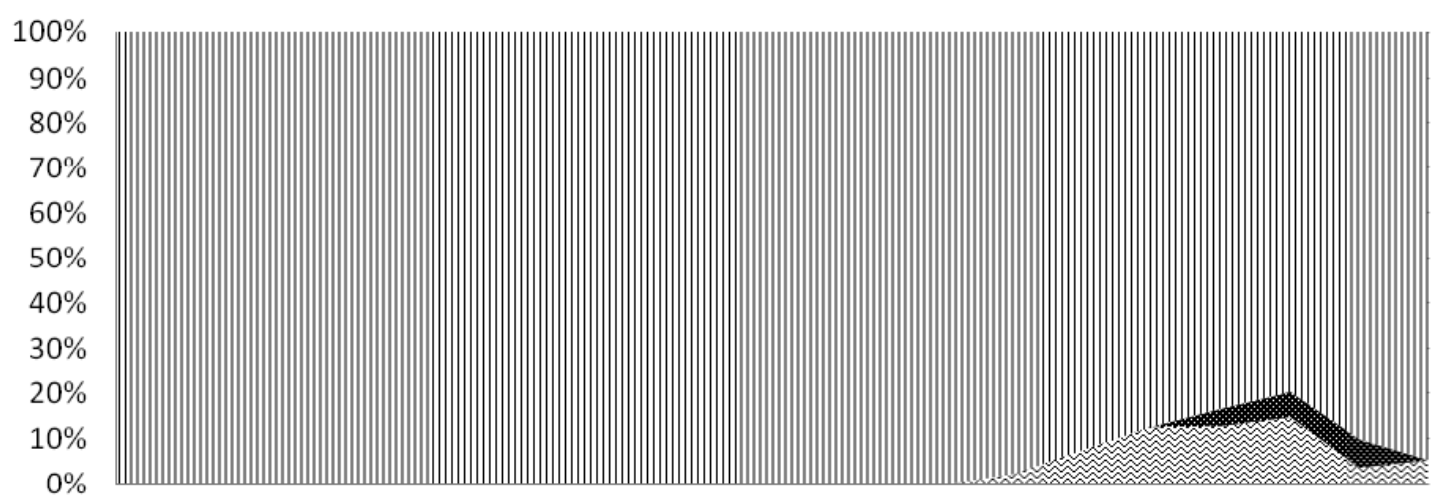

19901991199219931994199519961997199819992000200120022003200420052006200720082009

淠 GASTO de PDVSA 骂 GASTO de FONDEN III Gasto Estatal

Gráfico 8. Gasto social estatal y para-estatal en miles de BF de 2008. Venezuela (1990-2009). Fuente: elaboración propia en base a ONAPRE y BCV.

cer período se inicia en el año 2009, con la implementación de la Asignación Universal por Hijo, tras el conflicto abierto en 2008 en torno a la Ley $125^{4}$ y la derrota electoral que sufre el gobierno argentino en el mes de junio de 2009 (en las elecciones legislativas). En ese contexto, una de las vías de recomposición hegemónica implementadas por el régimen lleva al gasto en asistencia social a un nivel más elevado, pero ahora a través del rubro Previsión Social. Tanto el kirchnerismo como el chavismo recurren al aumento de gasto en momentos donde su hegemonía tambalea. Esta breve referencia plantea la necesidad de incorporar al análisis de las políticas sociales la dimensión de lucha clases, punto que en esta oportunidad no podremos desarrollar. ${ }^{5}$

En cuanto a las vías de canalización del gasto social en su conjunto, en Argentina se observa el aumento de la participación de las instancias provinciales y municipales en detrimento de la intervención nacional. La última década, junto con los primeros años de 1990, constituye uno de los momentos de mayor descentralización: el
40 por ciento del gasto se realiza vía instancias subnacionales. En el caso de Venezuela, si bien la descentralización en su momento más importante, en el año 2002, alcanza valores similares a los de Argentina, bajo el chavismo se observa una tendencia a la centralización del gasto en el gobierno nacional (administrando el 80 por ciento del mismo). Aunque, al mismo tiempo, se observa una tendencia a canalizar una parte del gasto por vías extraestatales, a través de empresas como PDVSA y de fondos especiales como el FONDEM. Tendencia que, sin embargo, como puede observarse en el gráfico 8 , no tiene un peso relativo importante.

Como síntesis de lo descrito en este apartado podemos afirmar que lo específico de la política social en el período estudiado reside en el crecimiento de la política asistencial. El gasto en Educación también crece, aunque puede implicar un cambio cualitativo no puede soslayar el hecho de que la escuela pierde en forma creciente su rol educativo y ha pasado a cumplir una función asistencial importante (De Luca, 2008). ${ }^{6}$

${ }^{4}$ El mismo tomó estado público como «conflicto del campo» y se desata con el anuncio presidencial de aplicar un nuevo sistema de retención a las exportaciones del sector agrario.

5 Para el caso de Argentina 2001-2007, véase Seiffer (2011).

${ }^{6}$ Cumple al mismo tiempo una función económica en la medida en que la escolarización disminuye la competencia con los trabajadores que están ofreciendo su fuerza de trabajo en el mercado y política en tanto opera sobre las cifras del desempleo, pues un estudiante que no busca trabajo «activamente», no es considerado parte de la PEA. 


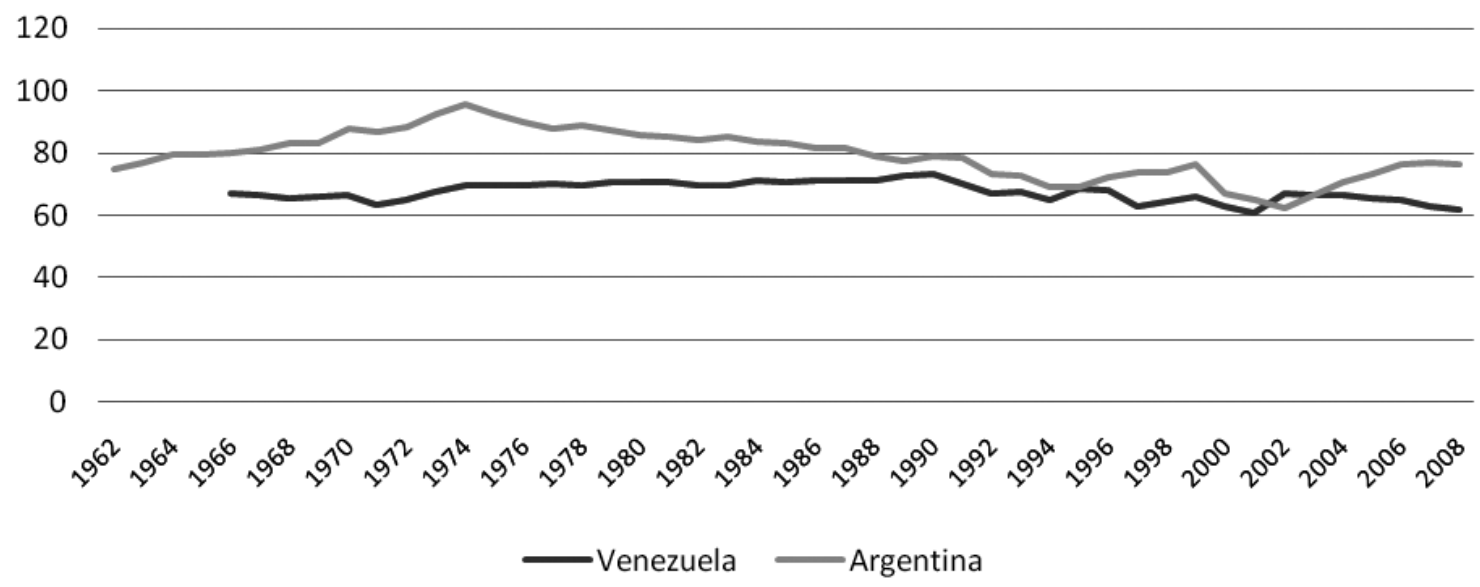

Gráfico 9. Asalariados como \% de la PEA. Argentina (1963-2009) y Venezuela (1976-2009). Fuente: elaboración propia en base a Ferreres, Baptista, INE, Graña y Keneddy.

El crecimiento en los rubros vinculados a la asistencia social muestra que la nueva fase de expansión del gasto no parece haber revertido la consolidación de un Estado asistencial (Grassi, 2003). Pero para avanzar en comprender el carácter de dicho gasto resulta fundamental dar cuenta de quiénes son sus beneficiarios.

\section{La consolidación de la población sobrante} Argentina y Venezuela son dos espacios nacionales donde las relaciones sociales capitalistas se han extendido plenamente. Ello se expresa en que la mayor parte de su población se ve obligada a vender su fuerza de trabajo como forma de obtener sus medios de vida. $^{7} \mathrm{El}$ carácter obrero de la mayor parte de su población se constata, en primer lugar, en los niveles de retribución salarial existentes. Para el caso de Argentina se observan niveles de retribución salarial sobre la población económicamente activa (PEA) que rondan el 80 por ciento, mientras en Venezuela se ubican entre el 60 por ciento y el 70 por ciento. Asimismo, como se ve en el gráfico 9, en ambos países se observa una tendencia a la caída de la participación de los asalariados sobre la PEA: en Argentina es una tendencia que se comienza a observar desde mediados de la década de 1970 y en Venezuela, a partir de los años 1990. En Argentina la tendencia se revierte a partir de
2003, ubicándose de todas maneras por debajo de los valores alcanzados históricamente.

Este hecho nos enfrenta a la siguiente pregunta: ¿está la clase obrera de estos países disminuyendo? Para responderla deberemos analizar una serie de elementos que dan cuenta del estado del mercado de trabajo en ambos países $\mathrm{y}$, al hacerlo, podremos encontrar a su vez elementos que explican los distintos niveles de retribución salarial en uno y otro.

Como dijimos, la caída en los niveles de retribución salarial podría expresar una disminución en la cantidad de asalariados reforzando la tesis de la desproletarización. Lejos de esto, se observa un crecimiento absoluto. No obstante, este movimiento, es acompañado por el crecimiento de la población económicamente activa (PEA) a una velocidad mayor, dando como resultado la caída en la tasa de retribución salarial.

Este aumento de la PEA, además de ser expresión del aumento de la población total, podría indicar el pasaje de la inactividad a la desocupación abierta; así como expresar un aumento absoluto y relativo de sujetos que participen del mercado sin verse forzados a vender su fuerza de trabajo. Veamos cada uno de estos elementos.

Mientras la tasa de desocupación en Argentina durante la década de 1970 oscila entre el 2

\footnotetext{
${ }^{7}$ Si bien mercantilización de la fuerza de trabajo y de las necesidades son distinguibles analíticamente son parte de un mismo y único proceso. Sobre la conformación de la fuerza de trabajo en mercancía, véase el capítulo «La llamada acumulación originaria», de El Capital (Marx, 2000).
} 


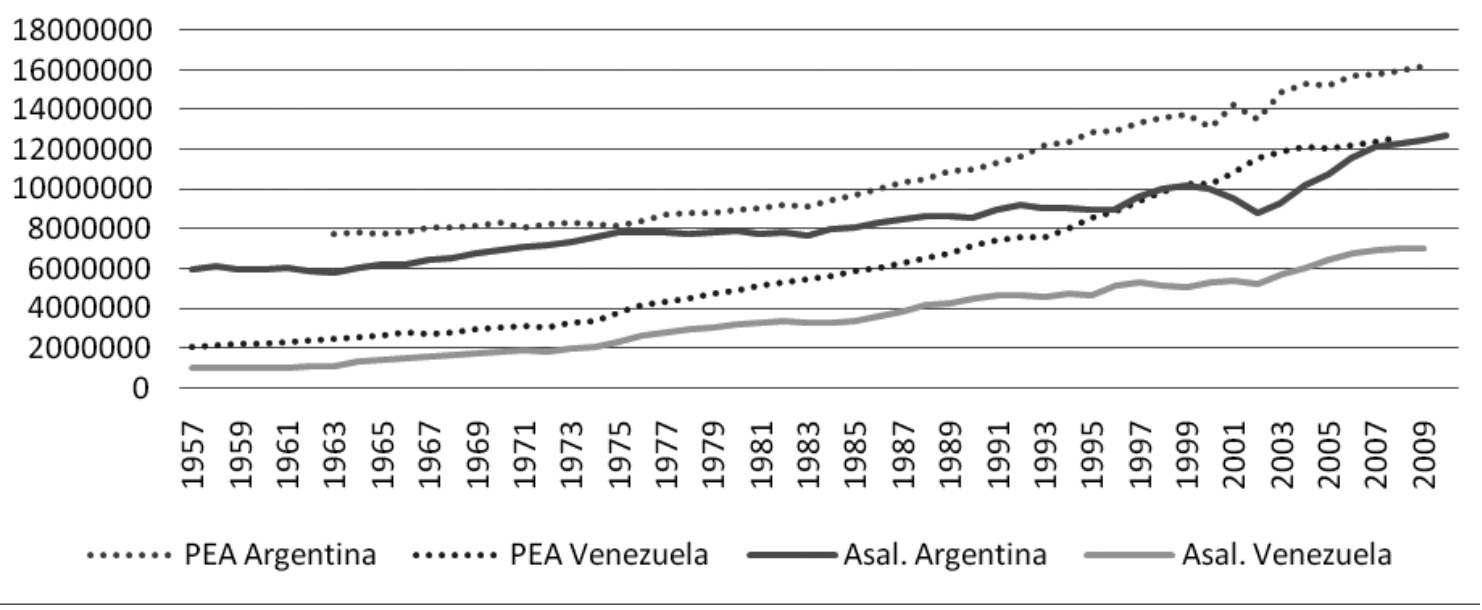

Gráfico 10. Población total, PEA y asalariados. Argentina y Venezuela (1957-2009). Fuente: elaboración propia en base a Ferreres, Baptista, INE, BCV, Graña y Keneddy e INDEC.

por ciento y el 5 por ciento, a partir de la década de 1980 sufre un ascenso permanente y que encuentra sus picos en el año 1995 primero y en el año 2003 después (alcanzando al 23 por ciento de la PEA). ${ }^{8}$ Hasta el año 2003, los momentos de disminución del desempleo sin embargo, no rompían el piso impuesto por el momento anterior a la expresión más cruda de la crisis. Desde entonces se observa una importante caída de la desocupación que se estanca a partir del año 2007. Venezuela, por su parte, hasta inicios de los años 1990, tenía tasas de desocupación más elevadas que las argentinas, ubicándose en torno al 7 por ciento durante los años 1960 y 1970. Con la crisis de mediados de los años 1970, las tasas de desocupación dan un salto encontrando su primer pico hacia el año 1985 con más de 13 por ciento de desocupación. Así como en Argentina, la disminución ubicó el piso en un nivel más alto y, a partir del año 1994, ha seguido un crecimiento importante que que ha encontrado su pico también en el año 2003. A pesar de este importante crecimiento, las tasas de desocupación venezolanas desde los años 1990 tienden a ubicarse por debajo de las de Argentina.

Uno de los elementos que pueden explicar este último factor es el peso relativo mayor que adopta el empleo público como fuente de ocupación en Venezuela respecto de Argentina. El peso de los asalariados públicos sobre el total es históricamente mayor en Venezuela. En Argentina se observa un aumento de su peso en los contextos de crisis y, en tanto el total de asalariados no disminuye, ${ }^{9}$ debe tratarse de una expansión del empleo público como forma de absorción de trabajadores desocupados. Tras el crecimiento que tiene lugar en el momento álgido de la crisis, tiende a mantenerse relativamente estable en valores similares a los de la primera parte de los años 1990. En el caso de Venezuela, durante la segunda parte de los años 1980 y primeros de los 1990, hay una tendencia a la disminución del empleo público sobre el total; a partir de entonces sufre un estancamiento relativo hasta el año 2001 y de ahí en adelante tiende a crecer superando los valores alcanzados históricamente.

Estos primeros datos ponen en evidencia que tanto Argentina como Venezuela son procesos de acumulación de capital que requieren de la producción normal de una población sobrante para sus necesidades inmediatas de acumulación; población que se presenta bajo la

\footnotetext{
${ }^{8} \mathrm{Si}$ se incorporan los datos de subocupación, la cifra asciende a más del 40 por ciento de la PEA.

${ }^{9}$ Excepto en el contexto agudo de la crisis de 2001-2003, cuando disminuye también la PEA, lo que hace suponer una población que pasa a estar inactiva porque no logra encontrar trabajo bajo ninguna condición.
} 


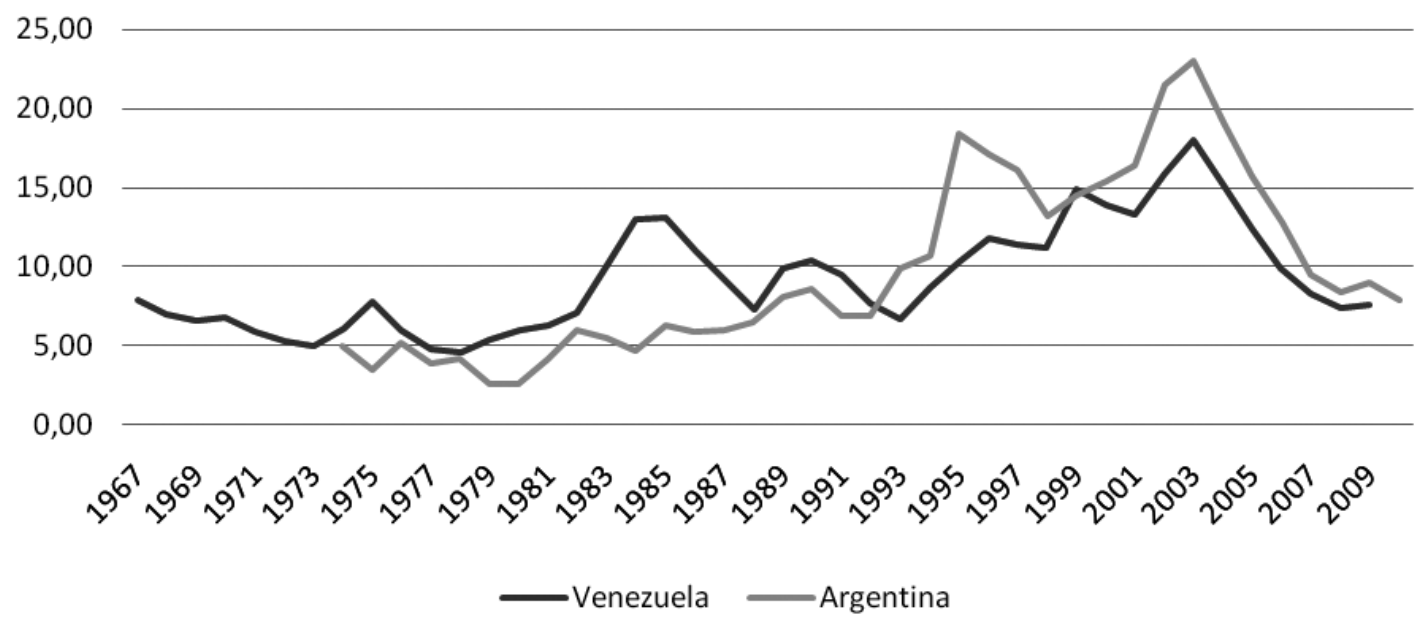

Gráfico 11. Tasa de desocupación. Argentina (1974-2009) y Venezuela (1967-2008). Fuente: elaboración propia en base a EPH-INDEC e INE.

forma de desocupación abierta y de expansión del empleo público improductivo.

El aumento del empleo estatal lleva a una nueva problemática: la existencia de población sobrante para el capital no solo dentro de los desempleados. Marx ya apuntaba esta situación cuando planteaba la existencia de distintas formas de sobrepoblación y establecía su forma estancada:

Constituye una parte del ejército obrero activo, pero su ocupación es absolutamente irregular, de tal modo que el capital tiene aquí a su disposición a una masa extraordinaria de fuerza de trabajo latente. Sus condiciones de vida descienden por debajo del nivel medio normal de la clase obrera y [es] esto, precisamente, lo que convierte a esa categoría en base amplia para ciertos ramos de explotación del capital. El máximo de tiempo de trabajo y el mínimo de salario la caracterizan (Marx, 2000, p. 801).

Así es que, tanto en Argentina como en Venezuela, existe una capa de la sobrepoblación relativa que se encuentra empleada. Esta corresponde a aquellas fracciones que venden su fuerza de trabajo a capitales que no tienen la productividad media, es decir que se trata de empresas que no logran valorizarse en condiciones normales y, por lo tanto, tienden a desaparecer en la competencia capitalista como resultado de la concentración y centralización. Pero aún sin desaparecer, para compensar su menor productividad estos capitales necesitan comprar la fuerza de trabajo por debajo de su valor. Se trata, por lo tanto, de una fracción de la clase obrera que aunque en apariencia se reproduce de forma normal, es parte de la población sobrante para el capital. El indicador más fuerte en este sentido es el gran descenso del salario real tanto en Venezuela como en Argentina que, pese a la recuperación económica, no logra alcanzar los niveles previos a dicha caída, pero que, además, está muy por debajo de los salarios en los países donde la clase obrera se reproduce en forma normal (Iñigo Carrera, 2007; Mateo Tomé, 2004). Tal y como puede observarse en el gráfico 12, el salario industrial en Venezuela y en Argentina pierde posiciones respecto del de Estados Unidos.

Esta caída salarial va de la mano del crecimiento de la informalidad laboral. El 40 por ciento de los trabajadores con empleo en Argentina están «en negro»: no tienen contrato de trabajo, aguinaldo, vacaciones pagadas y no cuentan tampoco con obra social ni el futuro beneficio de la jubilación. Asimismo, la evolución de los salarios reales permite observar que a partir de 1999, los salarios de los empleados en «blanco y en negro» se van distanciando; en promedio, durante los últimos 8 años, el salario no registrado no supera el 30 por ciento del salario registrado. La misma situación se observa en Venezuela en donde la informalidad en la última década ha alcanzado valores de entre el 43 por ciento y el 53 por ciento. 


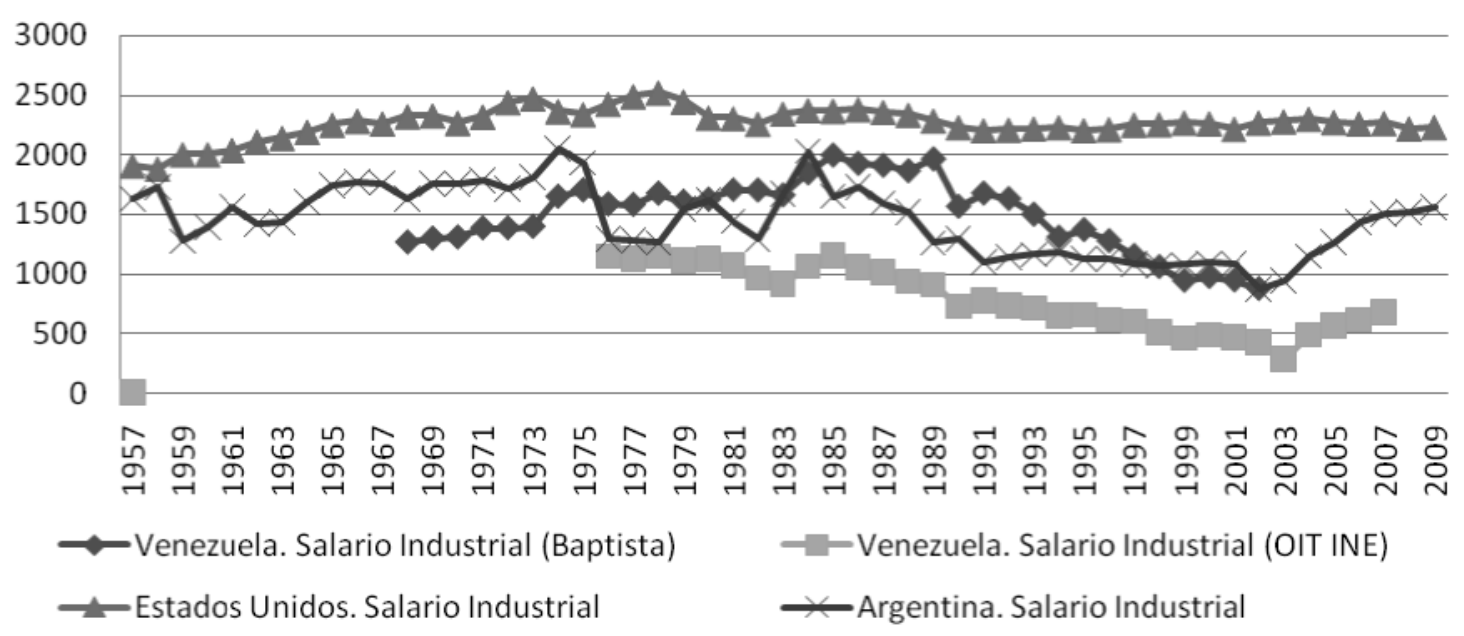

Gráfico 12. Salario industrial. Argentina (1957-2009), Venezuela (1957-2002 y 1974-2007) y Estados Unidos $(1957-2009)$. En PPA $(1993=100)$. Fuente: elaboración propia en base a fuentes varias.

Otro de los casos que corresponden a esta situación es el de los trabajadores por cuenta propia. Dicha categoría ha venido creciendo de forma ininterrumpida en Venezuela: en 1967 se contabilizaban 658.230 trabajadores por cuenta propia, en 1993 la cifra asciende a 1.768.923 y, hacia 2009, encontramos un total de 3.581.033 trabajadores. Entre 1968 e inicios de los años 1990 representan al 23 por ciento promedio de los ocupados, cifra que asciende al 31 por ciento promedio hacia 1999 y se estanca en el 30 por ciento durante la década chavista. En el caso de Argentina también se verifica la importancia del peso del «cuentapropismo» sobre la economía que representa, entre 1974 y 2009, un cuarto de la población ocupada del total del país. Son diversos los autores que reconocen la gran heterogeneidad que engloba la categoría «por cuenta propia» (Torrado, 1992; MTEySS, s.f.; Donaire, 2007; Bertranou y Maurizio, 2011). El problema es que las fuentes existentes no permiten identificar en forma directa si estos trabajadores independientes disponen o no de medios de producción. Algunos análisis hacen una diferenciación interna de la variable a par- tir de la categoría laboral (Donaire, 2007; Jiménez, 2011), destacando que al menos el 25 por ciento de los «cuentapropistas» pertenece a la clase obrera. Este grupo es el que más tiende a crecer en contextos de aumento del desempleo. En el caso de Venezuela, en ausencia de mejores fuentes para dar cuenta de la extracción de clase de la categoría censal «cuentapropista», tomamos el nivel de ingreso como indicador y lo que observamos es que el ingreso promedio de los «cuentapropistas» se encuentra, de manera sostenida, por debajo del ingreso de los asalariados. En tanto su ingreso promedio se ubica por debajo del ingreso medio del obrero, el trabajo por cuenta propia debe encubrir una forma de relación precaria con los poseedores de los medios de producción. Dado que este ingreso no alcanza para reproducir la fuerza de trabajo, difícilmente pueda ser suficiente como para reproducir un pequeño capital propio. Ante este cuadro es posible afirmar que la expansión del «cuentapropismo» es expresión de una masa de la población obrera que expulsa el capital del proceso inmediato de producción, en tanto le resulta sobrante. ${ }^{10}$

${ }^{10}$ En tanto la explotación es una relación entre clases y no entre individuos, ser expulsado del proceso inmediato de producción no es sinónimo de «exclusión». Quedar desocupado es la máxima expresión de la explotación que el capital total realiza al obrero, tomado colectivamente. Robert Castel incurre en el error de ver la explotación como una relación entre individuos, por ello le resulta posible afirmar que «los supernumerarios no son siquiera explotados» (Castel, 2006). 
Este cuadro, lejos de dar cuenta de una disminución de la clase obrera la da de la transformación de su estructura, que pasa de una forma relativamente homogénea a una más fragmentada, y a la expansión de una población sobrante para las necesidades medias de acumulación. Es este último elemento el que explica la masificación de la política de asistencia en ambos países. No se trata solo de los desocupados abiertos, sino también de aquellos que el capital no puede emplear en condiciones normales de productividad y, por lo tanto, paga la fuerza de trabajo por debajo de su valor, tal y como ocurre con el trabajo por cuenta propia.

\section{Política social y capital sobrante}

El fuerte crecimiento del PBI y el aumento del presupuesto de la política social, no solo en términos absolutos sino en relación al resto de la economía, no logra sacar a gran parte de la población beneficiaria de su condición de sobrante. Incluso las fracciones que salen de la situación de desempleo abierto y consiguen emplearse siguen sin poder reproducirse en condiciones normales. El gasto social, en particular el destinado a la asistencia, conlleva una mejora de las condiciones de vida de la clase obrera, pero no supone un cambio cualitativo en las condiciones de venta de su fuerza de trabajo. Pese a la apariencia de un cambio radical, la política social muestra que, lejos de ser autónoma, sus límites y potencialidades vienen establecidos por las condiciones de acumulación.

Si tomamos la evolución de la industria como indicador general, diferentes estudios han mostrado que la capacidad de los capitales que se acumulan en Venezuela y Argentina se encuentra muy por debajo de la media mundial en cuanto a productividad (Iñigo Carrera, 2007; Dachevsky, 2011). Argentina mantiene niveles históricos de productividad en torno al 60 por ciento de la industria norteamericana (excepto durante los años 1980 que se ubica levemente por debajo). En Venezuela, en cambio, hacia 1969 los ingresos generados por el obrero de la industria venezolana alcanzaron el 90 por ciento de los de Estados Unidos, pero desde 1970 en adelante se observa una caída persistente que coloca a Venezuela en niveles por debajo de los de Argentina.
Si la productividad argentina se encuentra históricamente rezagada en relación con la de Estados Unidos y la de Venezuela se desploma hasta niveles por debajo de los de Argentina, ¿qué elementos explican la menor productividad del trabajo y por qué logran una tasa de ganancia similar a la de Estados Unidos? La primera respuesta se halla en las transferencias que reciben del sector primario, fundamentalmente agrario en Argentina y petrolero en Venezuela. ${ }^{11}$ Venezuela, logró hasta la década de 1970 mejores condiciones que Argentina, debido a que la evolución del precio del petróleo fue más estable que la de otras materias primas. Pero hacia finales de esta década, la llamada «crisis del petróleo» implicó el colapso y la contracción de la economía venezolana. La segunda respuesta se encuentra en la posibilidad de pagar salarios por debajo de los que corresponden a la reproducción normal de los trabajadores sobre la base de la expansión de la población sobrante.

Estas fuentes de compensación son las que permiten la igualación de las tasas de ganancia y dan la apariencia de que Argentina y Venezuela son espacios nacionales donde los capitales se valorizan normalmente. Pero, aunque los precios de las mercancías agrarias y del petróleo hayan mejorado en la última década, el carácter específico de los procesos de acumulación de estos países no se ha transformado. Lejos de pasar a sostenerse sobre otras bases, lo que implicaría un verdadero cambio de orientación, la mayor disponibilidad de riqueza, debido al aumento de la renta agraria y petrolera, es la base de la expansión relativa que viven ambos países. El kirchnerismo y el chavismo expresan un momento del proceso de acumulación que sigue sosteniéndose sobre la base de la reproducción de capitales que resultan sobrantes desde el punto de vista general. El llamado «apoyo a la industrialización» no es más que la reproducción de esta forma de acumulación en un contexto de alza de los precios de las mercancías que logran exportar, lo que les permite apropiarse de grandes masas de riqueza social. Los procesos de estatización que se han llevado adelante son o bien procesos de rescate de capitales quebrados (por ejemplo, Aerolíneas Argentinas y SIDOR en Venezuela) o una nueva

${ }^{11}$ Sobre los mecanismos de transferencia de renta en Argentina, véase Iñigo Carrera (2007) y en Venezuela, Dachevsky (2011). 
fuente de compensación para la reproducción de la especificidad de los procesos de acumulación de capital, cuando la fuente normal de financiación tiende a estancarse (por ejemplo: Administradoras de Fondos de Jubilaciones y Pensiones en Argentina y PDVSA en Venezuela).

El crecimiento económico relativo que viven ambos países, producto de la expansión de la renta agraria y petrolera, no es la base de un nuevo «modelo» de crecimiento, sino la réplica de la forma histórica de reproducción de capital de estos países. Y a capital sobrante, población sobrante. Por este motivo, si bien la extensión de la política de asistencia conlleva la reproducción de amplios sectores obreros en mejores condiciones, en tanto no altera las bases materiales de la acumulación, no elimina su condición de sobrantes, simplemente lo pone en evidencia de forma inmediata. Lo que aparece como un quiebre no es más que el proceso potenciado por la enorme disponibilidad de riqueza social proveniente del sector primario en un contexto de alza de los precios de los commodities. $^{12}$

\section{Conclusiones}

$\mathrm{Si}$ históricamente la política asistencial ha cumplido en estos países un papel de alguna manera residual, el aumento de la población sobrante extiende su papel. Los estados de Argentina y Venezuela han tenido que hacer un esfuerzo por dar respuesta a este problema y ese esfuerzo tiende a hacerse cada vez más importante (incluyendo los años 1990, cuando el Estado supuestamente se habría retirado).
Las fuentes principales sobre la que se sostienen los procesos de acumulación de Venezuela y Argentina son la renta petrolera y agraria respectivamente. Su expansión, al tiempo que permite el crecimiento económico general, es la base de la expansión del gasto social y del gasto en asistencia social. En Argentina esta fuente de riqueza se combina, con énfasis en los momentos de estancamiento y crisis o, cuando esa riqueza pasa a ser insuficiente, con la toma de deuda, emisión de moneda o, como ha sucedido recientemente, recurriendo a nuevos fondos (por ejemplo, fondos de pensiones en Argentina).

En la medida en que no haya un cambio cualitativo de las bases sobre las que operan estos capitales, la expansión de la política social y de su componente asistencial, si bien mejora parcialmente las condiciones de vida de la población obrera y puede permitir la consolidación de gobiernos con fuerte apoyo popular, no es capaz de revertir su condición de fuerza de trabajo sobrante. En cuanto las fuentes extraordinarias de financiación se contraigan, se pondrá nuevamente a la luz la miseria general que provoca el capitalismo. En tal sentido, la apreciación generalizada de que estamos ante una nueva fase, en cuanto a política social de la mano de gobiernos que enfrentan al capital, se muestra como resultado de un recorte centrado en los aspectos cuantitativos de corto plazo, que no da cuenta de las tendencias históricas que expresan el agotamiento de las potencialidades del capital para contener al conjunto de la población obrera de dichos países.

\section{Referencias bibliográficas}

Aponte Blank, C. (2006). El gasto público social venezolano: sus principales características y cambios recientes desde una perspectiva comparada. Cuadernos del Cendes, 63 [on line]. Disponible en $\mathrm{http}: / / \mathrm{www}$. scielo.org.ve/scielo.php?script=sci_arttext\&pid=S1012-25082006000300005\& lng=es\&nrm=iso\%3E.ISSN201012-2508 (31 de octubre de 2011).

Aponte Blank, C. (2010). El gasto público social durante los períodos presidenciales de Hugo Chávez: 1999-2009. Cuadernos del Cendes, 73 [On Line]. Disponible en http://www.scielo.org.ve/ pdf/cdc/v27n73/art03.pdf

Baptista, A. (2006): Bases cuantitativas de la economía venezolana. 1830-2002, Caracas: Fundación Empresa Polar.

Baudino, V. (2009) El ingrediente secreto. Arcor y la acumulación de capital en la Argentina (1950-2002). Buenos Aires: Ediciones ryr.

Bertranou, F. y Maurizio, R. (eds). (2001). Trabajadores independientes, mercado laboral e informalidad en Argentina. Buenos Aires: OIT.

Castel, R. (2006) La metamorfosis de la cuestión social. Una crónica del salariado. Buenos Aires: Paidós. 
Dachevsky, Fernando. (2011). La renta de la tierra petrolera y sus efectos en la acumulación de capital. El caso venezolano (1970-2010). (Tesis de maestría inédita), Universidad de Buenos Aires, Facultad de Ciencias Económicas.

De Luca, R. (2008). Brutos y Baratos. Descentralización y privatización en la educación argentina (1955-2001). Buenos Aires: Ediciones ryr.

Donaire, R. (2007). Quienes son los «trabajadores por cuenta propia» (Argentina 1980/2001). Lavboratorio. Estudios sobre cambio estructural y Desigualdad Social, 20. [On Line]. Disponible en http://lavboratorio.fsoc.uba.ar (27 de febrero de 2011).

Ferreres, O. (2011). Dos siglos de economía argentina. Buenos Aires: Fundación norte y sur.

Graña, J. y Kennedy, D. (2010). Salario real, costo laboral y productividad argentina 1947-2006. (Documento $\mathrm{n}^{\mathrm{o}}$ 12). Buenos Aires: Instituto de Investigaciones Económicas de la Facultad de Ciencias Económicas de la Universidad de Buenos Aires y por el Centro de Estudios sobre Población, Empleo y Desarrollo.

Grassi, E. (2003). Políticas y problemas sociales en la sociedad neoliberal. La otra década infame (I). Buenos Aires: Espacio.

Iñigo Carrera, J. (2007). La formación económica de la sociedad argentina. Volumen I. Renta agraria, ganancia industrial y deuda externa. 1882-2004. Buenos Aires: Imago Mundi.

Jiménez, M. (2011). Caracterización del trabajo independiente en Argentina. En F. Bertranou, y R. Maurizio, (eds.), Trabajadores independientes, mercado laboral e informalidad en Argentina. (pp. 39-94). Buenos Aires: OIT.

Kornblihtt, J. (2008). Crítica del marxismo liberal. Competencia y monopolio en el capitalismo argentino. Buenos Aires: Ediciones ryr.

Marx, Karl. (2000). El Capital. El proceso de producción del capital, Tomo I, Vol. 3. México: Siglo XXI.

Mateo Tomé, J. P. (2004, marzo, 25-27). La distribución primaria de la renta en Venezuela: análisis de la relación salarial (1979-96). En IX Jornada de Economía Crítica [On line]. Disponible en http://www.ucm.es/info/ec/jec9/pdf/A07\%20-\%20Mateo\%20Tom\%E9,\%20Juan\%20Pablo.pdf

Mateo Tomé, J. P. (2010). La evolución de los salarios en Venezuela. Una década de contrastes. Boletín Económico de ICE, 2994. [On Line]. Disponible en http://www.revistasice.com (26 de octubre de 2011).

Ministerio de Trabajo, Empleo y Seguridad Social. (s.f.). La heterogeneidad del cuentapropismo en la Argentina actual. Una propuesta de análisis y clasificación. (Documento $\mathrm{n}^{\circ} 5$, Trabajo, ocupación y empleo. Especialización productiva, tramas y negociación colectiva). Buenos Aires: MTEySS.

Reyes, O. (2006). Sobre el socialismo del Siglo XXI en Venezuela. Stockholm Review of Latin American Studies, 1.

Rodríguez Rojas, P. (2010). Venezuela: del neoliberalismo al socialismo del siglo XXI». Política y Cultura, 34. [On Lines]. Disponible en http://www.scielo.org.mx/scielo.php?pid=S0188$77422010000200009 \&$ script $=$ sci_arttext (31 de octubre de 2011).

Sartelli, E. (2007). La plaza es nuestra. El Argentinazo a la luz de la lucha de la clase obrera en la Argentina del siglo XX. Buenos Aires: Ediciones ryr.

Sartelli, E. (dir.). (2008). Patrones en la ruta. El conflicto agrario y los enfrentamientos en el seno de la burguesía, marzo-julio de 2008. Buenos Aires: Ediciones ryr.

Seiffer, T. (2011). La lucha de clases y la política de asistencia en Argentina, 2002-2007. En M. Mallardi, L. Madrid y A. Oliva (comp.), Cuestión social, reproducción de la fuerza de trabajo y políticas de asistencia (pp. 43-69). Tandil: Carrera de Trabajo Social, Universidad Nacional del Centro de la Provincia de Buenos Aires.

Torrado, S. (1992). Estructura social de la Argentina 1945-1983. Buenos Aires: Ediciones de la Flor. 